\title{
Acute Heart Failure in a Healthy Young Patient after Severe Carbon Monoxide Poisoning
}

\author{
Christos E Lampropoulos $^{1}$, Evagelia Sklavou ${ }^{2}$, Charalampos Anastogiannis $^{2}$, Vasiliki Papanikolaou ${ }^{1}$, \\ Dimitris Tsilivarakis ${ }^{1}$, Vasilis Vasiliou ${ }^{1}$, Sofia Vakali ${ }^{1}$ \\ ${ }^{1}$ Department of Internal Medicine, Panarcadian General Hospital, Tripoli, Greece \\ ${ }^{2}$ Intensive Care Unit, General Hospital of Pyrgos, Pyrgos, Greece
}

Received: 21/10/2019

Accepted: 04/11/2019

Published: $13 / 12 / 2019$

How to cite this article: Lampropoulos CE, Sklavou E, Anastogiannis C, Papanikolaou V, Tsilivarakis D, Vasiliou V, Vakali S. Acute failure in a healty young patient after severe carbon monoxide poisoning. EJCRIM 2019;6: doi:10.12890/2019_001340.

Conflicts of Interests: The Authors declare that there are no competing interest

Acknowledgements: We would like to thank Dr loanna Papaioannou, Dr Athanasio Kantouni, Dr Anna Sidiropoulou, Dr Maria Avgoulea and Dr Afroditi Roumpou for their contribution in patient's management.

This article is licensed under a Commons Attribution Non-Commercial 4.0 License

\section{ABSTRACT}

Introduction: Carbon monoxide (CO) poisoning may cause severe cellular hypoxia.

Materials and methods: A 28-year-old male presented reduced levels of consciousness and dyspnoea after CO exposure. Clinical examination revealed tachypnoea, bilateral rales, dilated jugular veins and confusion. Troponin I, lactate and carboxyhaemoglobin levels were increased. Thoracic X-ray depicted pulmonary oedema and an echocardiogram, severe heart failure (HF; EF<25\%). He was intubated due to clinical deterioration.

Results: He remained intubated for 5 days with excellent improvement of left ventricular function (EF $>55 \%$ ). He was discharged 1 week later with full recovery.

Discussion: Acute HF is a rare serious complication of $\mathrm{CO}$ poisoning, even in healthy young individuals.

\section{LEARNING POINTS}

- $\quad$ Acute $\mathrm{CO}$ poisoning is an emergent condition necessitating prompt diagnosis and treatment.

- Neurologic and cardiovascular manifestations are the most worrying complications, which may leave permanent sequelae.

- Acute heart failure is a rare complication of CO poisoning, characterized by regional or global left ventricular wall hypokinesia

\section{KEYWORDS}

Carbon monoxide poisoning, acute heart failure, echocardiography, intensive care unit, hyperbaric oxygen therapy

\section{INTRODUCTION}

Carbon monoxide (CO) is a tasteless, odourless, gaseous pollutant generated by incomplete combustion of carbonaceous material. Gasolinepowered heat generators, indoor use of charcoal briquettes or exposure to CO from fire or intentionally as a suicide attempt are common aetiologies of toxicity. Almost 1,300 deaths are attributed to CO exposure annually in the United States. Even short-term exposure to low $\mathrm{CO}$ levels has been implicated in impaired central nervous system function, coronary heart disease (CHD), arrhythmias and heart failure (HF), especially in the elderly and in patients with pre-existing disease.

A case of a healthy young patient with severe $\mathrm{HF}$ as a consequence of acute $\mathrm{CO}$ poisoning is described here. 


\section{CASE DESCRIPTION}

A 28-year-old male was transferred to the Emergency Department (ED) with reduced levels of consciousness and reported seizures after the use of charcoal briquettes for heating all night. His medical history was free (no medication, smoking habits or alcohol intake).

Clinical examination revealed tachypnoea [respiratory rate $(R R)=30$ breaths/min], tachycardia (110 bpm), diffuse bilateral rales on lung auscultation and dilated jugular veins. Arterial pressure (AP), temperature, cardiac auscultation, abdominal palpation and oxygen saturation $\left(\mathrm{sO}_{2}\right)$ were normal (he was transferred with oxygen administration). No signs of peripheral pitting oedema were noticed. Neurologically, the patient was severely confused with a reduced Glasgow Coma Scale score (GCS=9, pain localization, concurrent eye-opening, incomprehensible sounds) and no focal neurologic signs or neck stiffness.

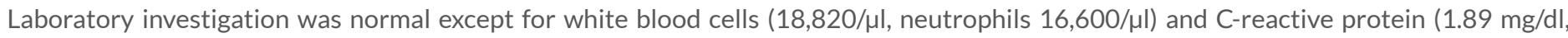
normal $<0.7 \mathrm{mg} / \mathrm{dl}$ ). Initial arterial blood gas analysis (50\% oxygen) showed sO $=99.7 \%, \mathrm{pO}_{2}=213 \mathrm{mmHg}, \mathrm{pCO}_{2}=35.1 \mathrm{mmHg}, \mathrm{pH}=7.431$, lactate $=2.9 \mathrm{mmol} / \mathrm{l}, \mathrm{HCO}_{3}=23 \mathrm{mmol} / \mathrm{l}, \mathrm{COHb}=29.5 \%$. An electrocardiogram (ECG) showed no ischaemia. Thoracic X-ray depicted diffuse, bilateral infiltrates of the lower lung fields, compatible with pulmonary oedema (Fig. 1A). Brain computed tomography (CT) did not show cerebral oedema or focal lesions. An echocardiogram revealed severe HF [ejection fraction (EF) <25\%] with diffuse, global hypokinesia and a dilated inferior vena cava with reduced respiratory fluctuation. High-sensitivity troponin I was $124 \mathrm{ng} / \mathrm{l}$ (<29 ng/l), whereas the other cardiac biomarkers were normal. Four hours later (while intubated), lactate was $1.83 \mathrm{mmol} / \mathrm{l}$ and $\mathrm{COHb} 2.5 \%$.

During his evaluation in the ED, the patient had a dramatic deterioration ( $\mathrm{AP}=75 / 50 \mathrm{mmHg}, \mathrm{RR}=50 \mathrm{breaths} / \mathrm{min}, \mathrm{sO} 2=80 \%$ despite $100 \%$ oxygen rate) and became comatose. He was intubated and transferred to the Intensive Care Unit (ICU). His appointment for emergent hyperbaric oxygen therapy (HOT) at the National Center (160 km away) was cancelled because of his unstable condition. The patient remained in the ICU for 5 days and was haemodynamically stable after the first day (without norepinephrine administration). A second brain $\mathrm{CT}$ confirmed the absence of oedema. He presented fever up to $38^{\circ} \mathrm{C}$, which subsided on the third day after initiation of meropenem $(2 \mathrm{~g} \times 3)$ and linezolid $(600 \mathrm{mg} \times 2)$. An echocardiogram showed excellent improvement of left ventricular contractility $(E F>55 \%)$. Troponin I levels were within normal limits.

The patient gradually regained consciousness after the fourth day, without memory problems or other neurologic deficits. He was transferred to the Internal Medicine Department for completion of treatment and rehabilitation. He was discharged 1 week later with full recovery (Fig. 1B). Neurologic and psychiatric evaluation 1 month later was normal.

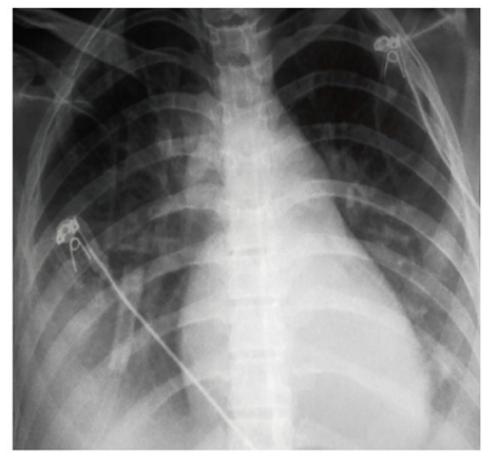

$\mathbf{A}$

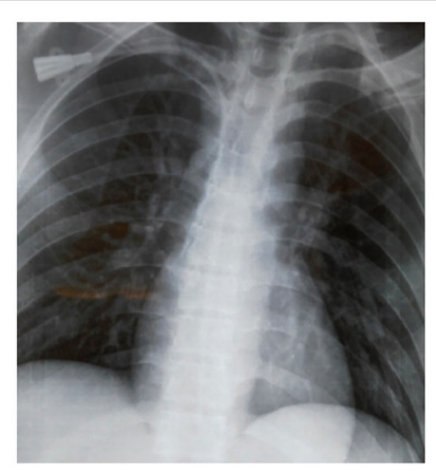

$\mathbf{B}$
Figure 1. Bilateral infiltrates of the lower lung fields, compatible with pulmonary oedema, during the initial episode of acute heart failure (A, supine position), with excellent improvement before discharge (B, upright position)

\section{DISCUSSION}

Cellular hypoxia after $\mathrm{CO}$ exposure is caused by disturbances of oxygen delivery. CO reversibly binds haemoglobin (230-270 times more powerfully than oxygen) and cardiac myoglobin with even greater affinity. Additionally, CO causes a leftward shift of the oxyhaemoglobin dissociation curve, further decreasing oxygen availability. However, impaired oxygen delivery does not sufficiently explain the extent of toxicity. CO may cause direct cellular damage involving immunological or inflammatory mechanisms (peroxynitrite production, reactive oxygen species, lipid peroxidation, mitochondrial oxidative stress, nitric oxide free radicals, platelet activation). The CO half-life is 3-4 hours. $100 \%$ oxygen reduces this to $30-90$ minutes and HOT ( 2.5 atmospheres, $100 \%$ oxygen) to $15-23$ minutes. 
The most profound impact is noticed on organs with the highest oxygen requirements (brain, heart). Symptoms typically begin with headaches $(\mathrm{COH} b=10 \%)$, progressing to memory problems, confusion, hallucinations, abdominal discomfort (pain, vomiting), visual disturbances and seizures, leading to coma or even death (levels up to 50-70\%). Patients with $\mathrm{CHD}$ may experience angina or arrhythmias with $\mathrm{COHb}$ levels of just 5-10\%, whereas at higher levels even young patients develop myocardial depression ${ }^{[1,2]}$. The most common electrophysiologic abnormality is disruption of repolarization and prolongation of the QT interval. Since 1928 (the first recorded case of angina), many reports, even those from young children, have documented the influence of $\mathrm{CO}$ on coronary vessels. HF and pulmonary oedema are an unusual complication, mainly in patients with pre-existing disease. Myocardial dysfunction may present as regional wall motion abnormality or global left ventricular dysfunction. Lee et al. presented a case of left ventricular dysfunction with concomitant intraventricular thrombus and Swank et al., a case of reversible acute $\mathrm{HF}^{[3,4]}$. Yanir et al. reported two previously healthy young patients with cardiogenic shock, whereas Kuniavsky et al., a young patient with $\mathrm{HF}$ and complete recovery after $\mathrm{HOT}^{[5,6]}$.

Physical examination is of limited diagnostic value as findings are non-specific. Inhalation injury or burns should always alert the clinician to the possibility of $\mathrm{CO}$ exposure. Cherry-red skin, though rare, has been considered a sign of CO poisoning. Ophthalmologic examination may reveal bright-red retinal veins (a sensitive early sign) or papilloedema.

Diagnosis of acute CO poisoning must be confirmed by elevated COHb levels (3-4\% in non-smokers, $10 \%$ in smokers), which may be reduced due to prior oxygen administration or a prolonged period since exposure. In patients with severe poisoning, ECG and cardiac biomarkers should be obtained. Elevated troponin I levels indicate cardiomyopathy or myocardial ischaemia, which is a predictor of mortality. Increased lactate levels are a severity index and correlate with neurologic outcome (levels $>10 \mathrm{mmol} / \mathrm{l}$ may indicate concomitant cyanide poisoning). A brain CT scan may reveal cerebral oedema and/or focal low-density lesions (typically in the basal ganglia), which may predict permanent neurologic sequelae. Magnetic resonance imaging, diffusion-weighted and diffusion tensor imaging are more accurate and can visualize pathologic changes in the early stages. A late finding is diffuse cerebral atrophy due to neuronal necrosis and apoptosis.

HOT contributes to prompt elimination of $\mathrm{COHb}$ and may diminish permanent cognitive sequelae or mortality. Selection criteria (regardless of the $\mathrm{COHb}$ levels) include coma, cardiac ischaemia, focal neurologic deficits or neuropsychiatric disorders. Treatment complications may be decompression sickness, sinus and ear barotrauma, seizures, pneumothorax, gas embolism or visual disturbances.

Despite aggressive treatment, many survivors (up to 19-37\% after 6 years of poisoning) are at risk for permanent neurologic and psychiatric complications (memory loss, irritability, movement or speech disorders, depression, psychosis, suicidal tendency). Mortality is significant, even in small children, and depends on $\mathrm{COHb}$ levels, encephalopathy, cardiomyopathy, duration of cardiopulmonary arrest, delayed treatment and co-morbidities.

\section{CONCLUSION}

Acute HF is a rare, serious but potentially reversible complication of CO poisoning. Aggressive treatment, and particularly, pre-hospital supportive care and urgent transfer to the hospital are life-saving.

\section{REFERENCES}

1. Garg J, Krishnamoorthy P, Palaniswamy C, Khera S, Ahmad H, Jain D, et al. Cardiovascular abnormalities in carbon monoxide poisoning. Am J Ther 2018;25:e339-e348.

2. Henry CR, Satran D, Lindgren B, Adkinson C, Nicholson Cl, Henry TD. Myocardial injury and long-term mortality following moderate to severe carbon monoxide poisoning. JAMA 2006;295:398-402.

3. Lee SJ, Kang JH, Kim NY, Baek IW, Park MY, Shim BJ, et al. A case report of carbon monoxide poisoning induced cardiomyopathy complicated with left ventricular thrombus. J Cardiovasc Ultrasound 2011;19:83-86.

4. Swank G, Jain AC, Morise AP, Schmidt S. Carbon monoxide poisoning: a case report of reversible cardiomyopathy. W V Med J 2004;100:228-231.

5. Yanir Y, Shupak A, Abramovich A, Reisner SA, Lorber A. Cardiogenic shock complicating acute carbon monoxide poisoning despite neurologic and metabolic recovery. Ann Emerg Med 2002;40:420-424.

6. Kuniavsky M, Bechor Y, Leitman M, Efrati S. Carbon monoxide poisoning in a young, healthy patient: a case study of heart failure recovery after hyperbaric oxygenation treatment. Intensive Crit Care Nurs 2018;47:85-88. 